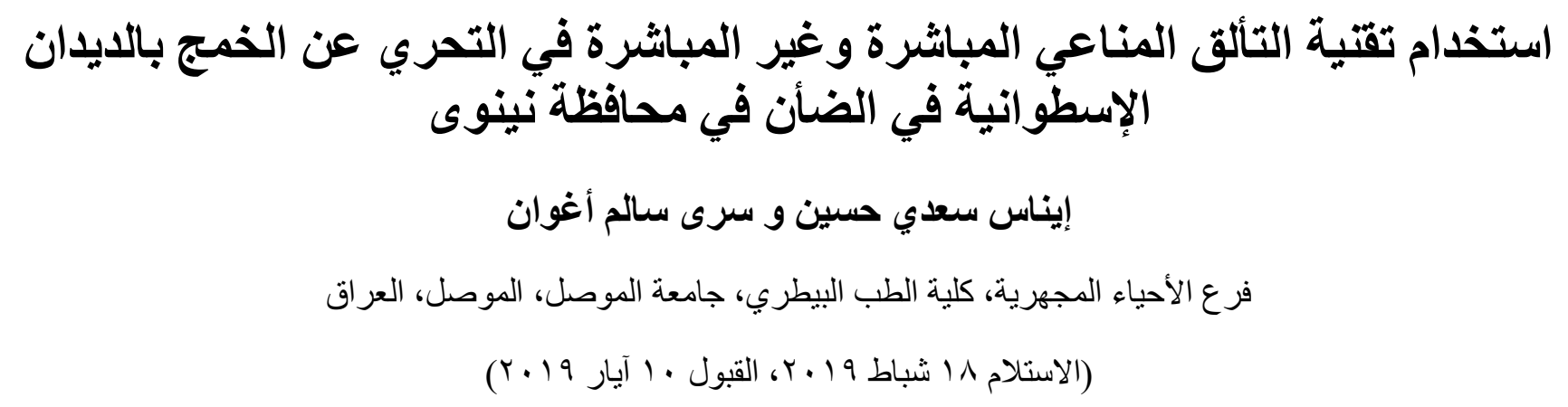

تضمنت هذه الدراسة إجر اء مقارنة بين طريقتين مختلفتين من طرق التشخيص الحديثة وذللك لتشخيص الخمج بالديدان الإسطو انية،

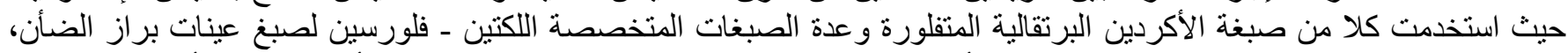

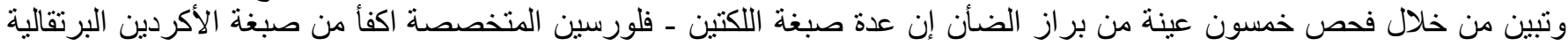

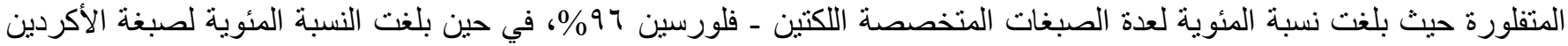

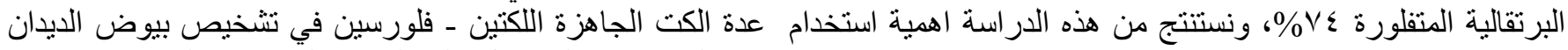

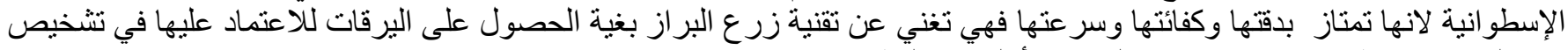

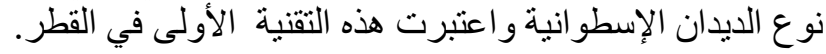

\title{
Uses of direct and indirect immuno-fluorescent techniques for demonstration of nematodes infection in sheep in Nineveh governorate
}

\author{
E.S. Hussein ${ }^{1}$ and S.S. Aghwan ${ }^{2}$ \\ Department of Microbiology, College of Veterinary Medicine, University of Mosul, Mosul \\ 1enassaadi5577@gmail.com, 를.s.s.aghwan@gmail.com
}

\begin{abstract}
This study was conducted for comparison between two different modern methods for the diagnosis of the nematode worms' infection in sheep we used acridine orange fluorochrome and specific staining kit in fecal samples. We examined 50 sheep fecal samples; the total infection rate was about $96 \%$ when was used lectin - fluorescein kit while the total infection rate was $74 \%$ when we used acridine orange fluorochrome technique. In conclusion of this study indicated that lectin - fluorescein kit is more effective than acridine orange fluorochrome in the diagnosis of nematode worms' eggs. Also, we conclude from this study the importance of using fluorescence - lectin kit technique in the diagnosis of nematodes eggs because they are distinguished by their speed, efficiency and accuracy, as they compare with the fecal culture technique to cultivate the larvae from eggs in order to diagnosed the type of nematodes eggs, the lectin - fluorescein kit technique is the first in Iraq.
\end{abstract}

Keywords: Indirect, Direct, immunofluorescent, Lectin - Fluorescein Kit Available online at http://www.vetmedmosul.com, (C) 2020, College of Veterinary Medicine, University of Mosul. This is an open access article under the CC BY 4.0 license (http://creativecommons.org/licenses/by/4.0/).

الحيو انات في مختلف أنحاء العالم ( (1) استخدمت صبغة الأكردين المقدمة

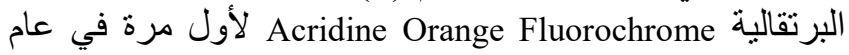

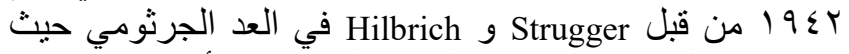
ترنبط هذه الصبغة بالحامض النووي المنقوص الأوكسجين في الني

يعد خمج الضأن بالديدان الإسطوانية من الأمراض المهئة

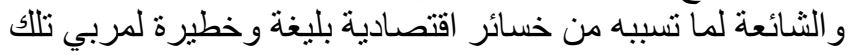




\section{صبغة الأكردين البرتقالية}

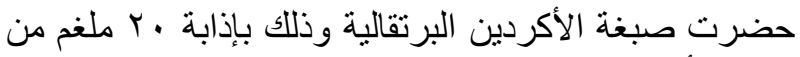

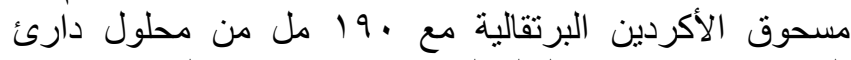

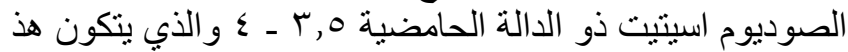

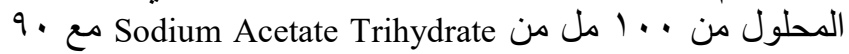
مل من حامض الهيدروكلوريك. مل وضعت مسحات البراز المثبتة

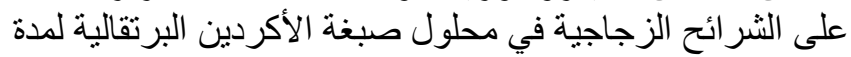

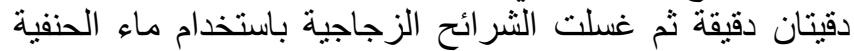

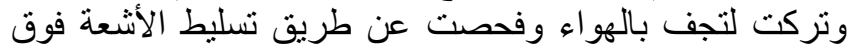

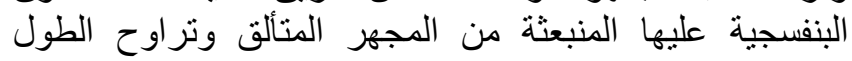

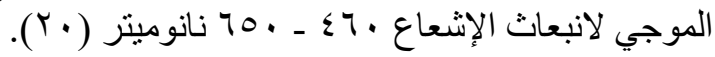

\section{عدة صبغة اللكتين ـ فلورسين المتخصصة}

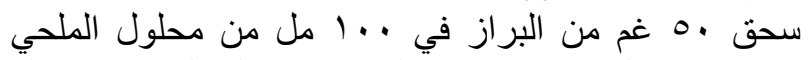

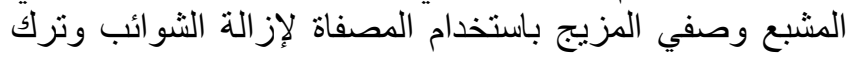

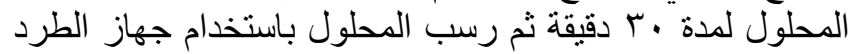

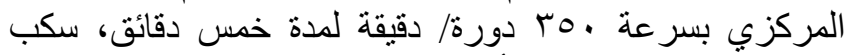

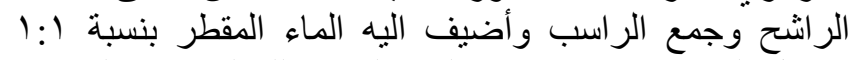

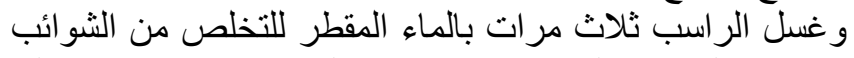

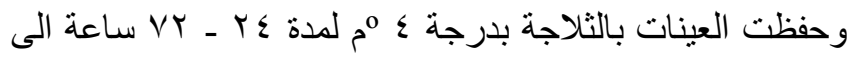

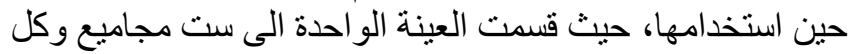

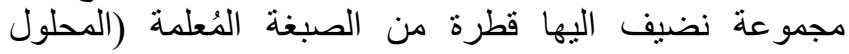

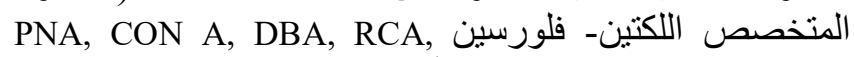

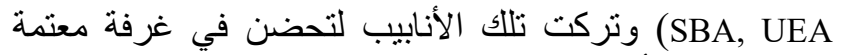

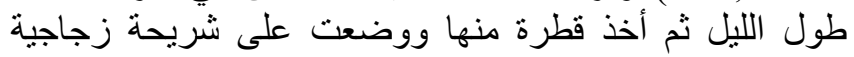

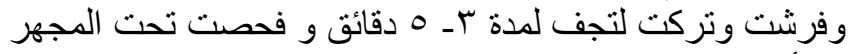

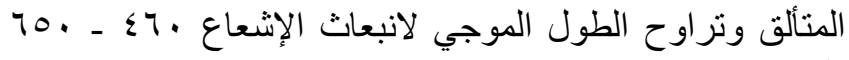

نانومتر (Y) (Y) (1)

\section{النتائج}

تبين من خلال فحص الثرائح الزجاجية لمسحات البراز

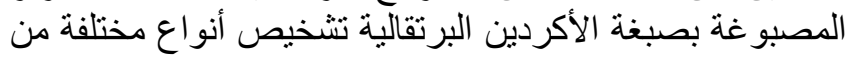

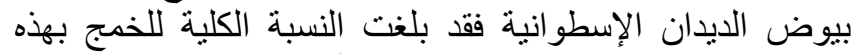

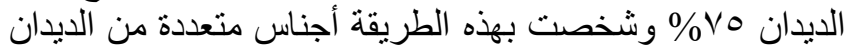

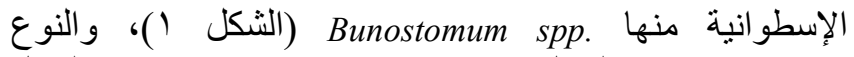
Ostertagia spp. Trichuris ovis r)، والجنس Trichostrongylus spp.

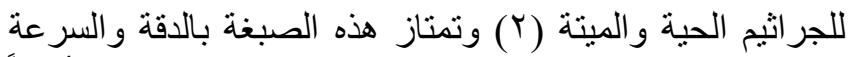

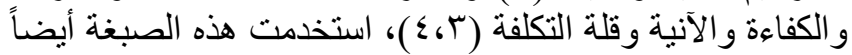

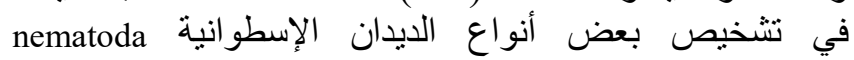

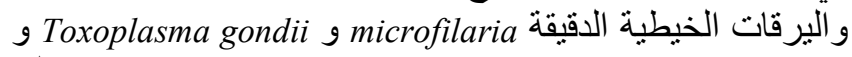
. Babesia spp. و Trypansoma spp.

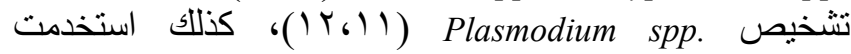
Suleiman

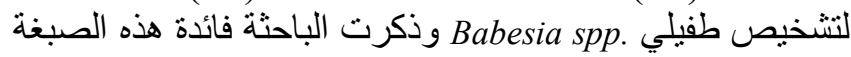

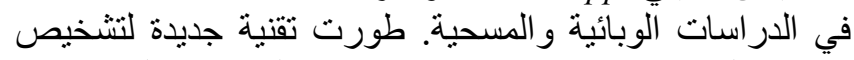

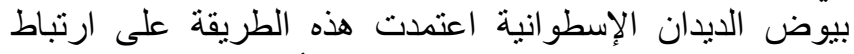

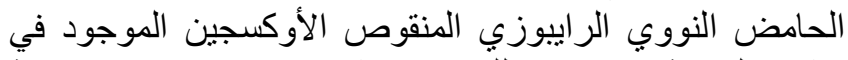

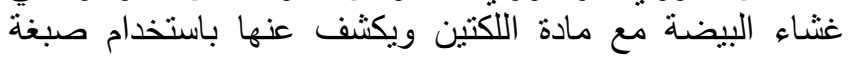

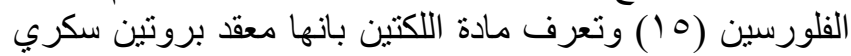

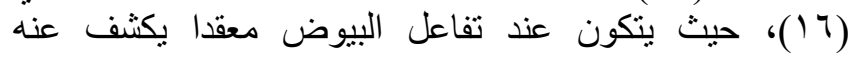

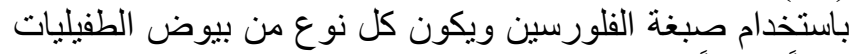

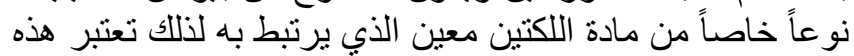

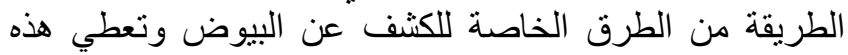

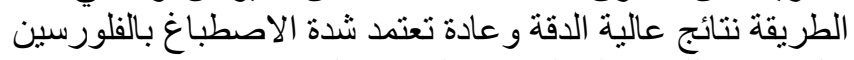

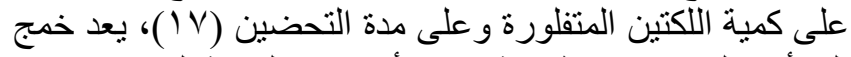

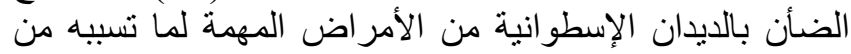

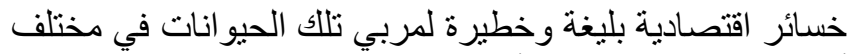

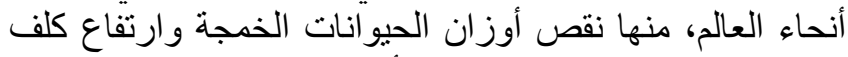

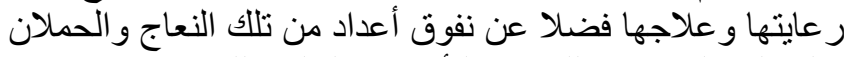
و المواليد الحديثة كذللك من التأثيرات السلبية للديدان اعندان الإسطوانية الإنية

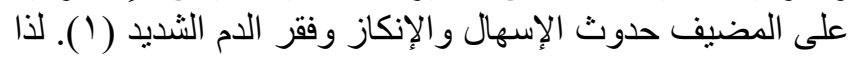

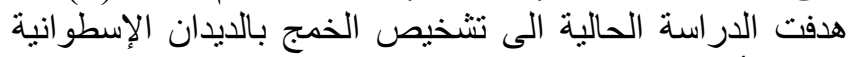

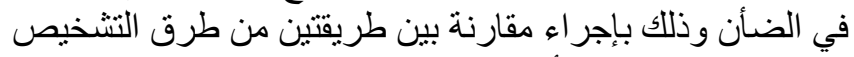

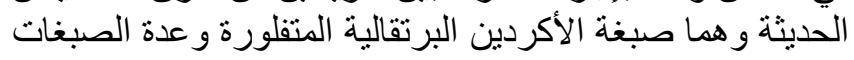

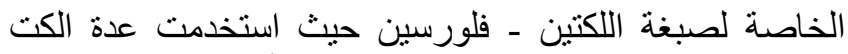
الجاهزة لتصبيغ بيوض الديدان الاسطو انية لأول مرة في القطر.

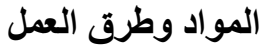

تحضير الثرائح الزجاجية

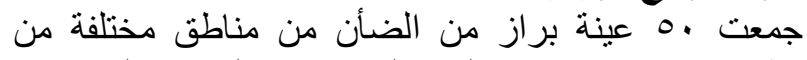
محافظة نينوى منها (كوكجلي، الرحمانية، تلكيف، الرئ، الحمدانية،

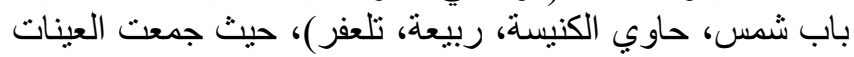

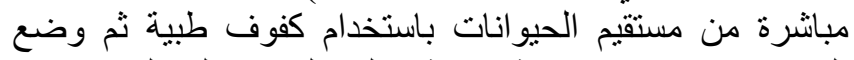

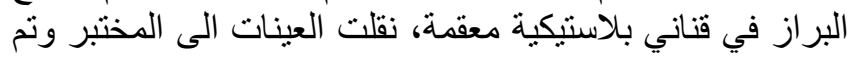

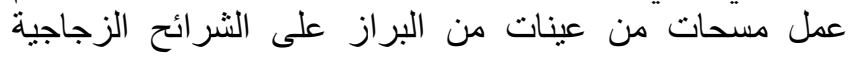

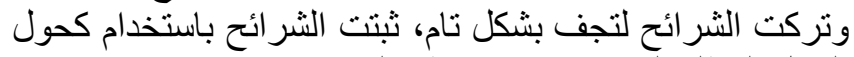

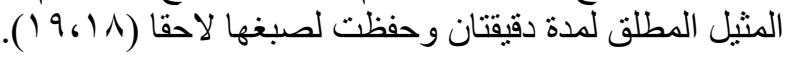


يرتبط بدوره بأنواع متخصصة من بيوض الديدان الإسطوانية

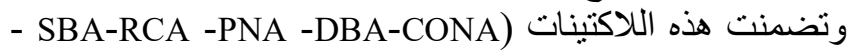
WGA -UEAI

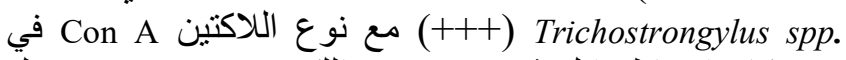

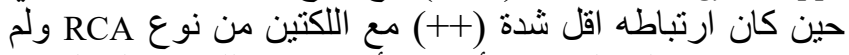

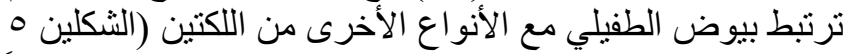

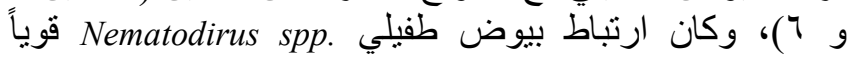

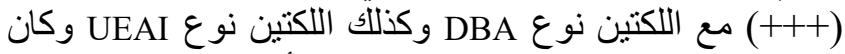

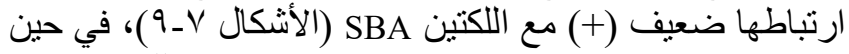

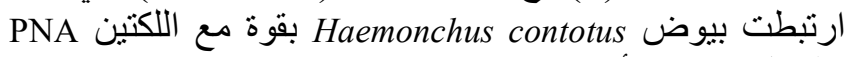

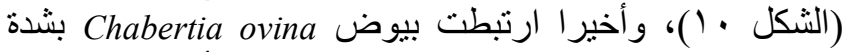

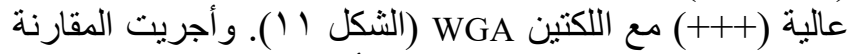
الإحصائية بين طريقة التصبيغ بالأكردين البرتقالية وطريقة

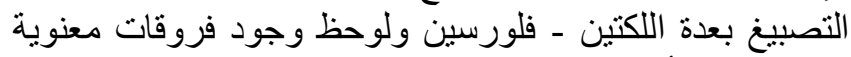

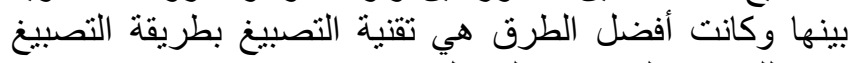
بعدة اللكتين - فلورسين (الجدول بان ).

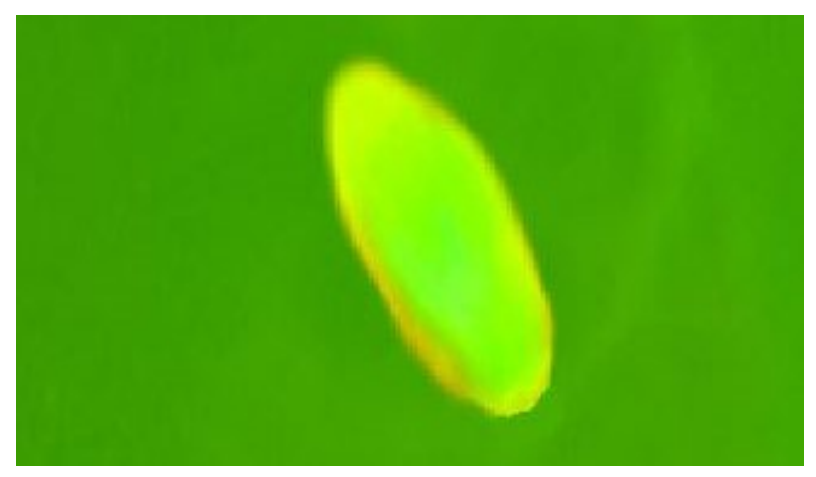

الشكل §: يوضح بيضة .Trichostrongylus spp باستخدام صبغة

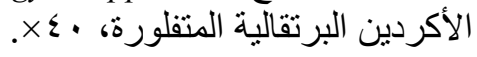

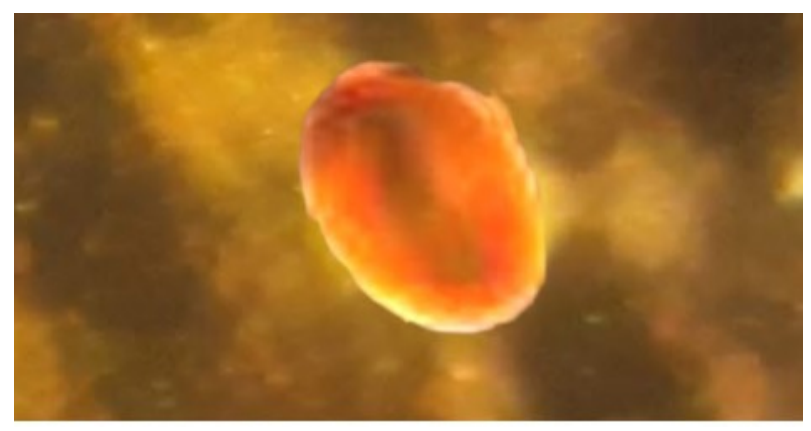

الثكل ๑: بيضة (PNA) . (Prichostrongylus spp.

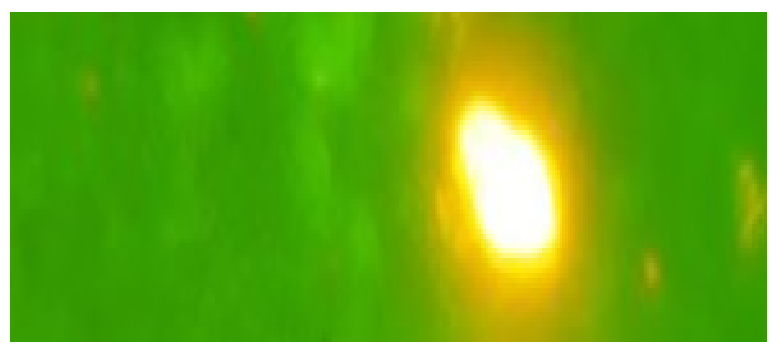

الثكل 1: يوضح بيضة .Bunostomum spp. باستخدام صبغة

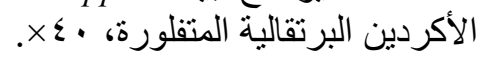

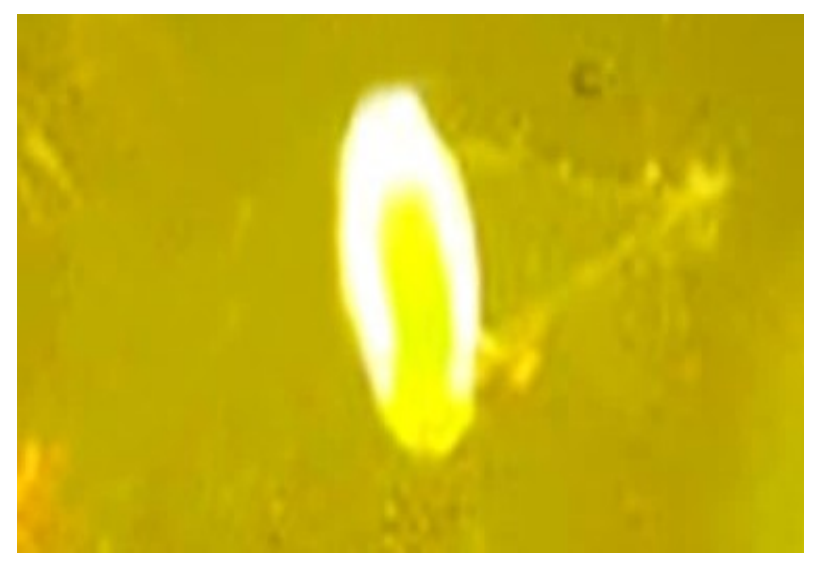

الثكل r : يوضح بيضة Trichuris ovis باستخدام صبغة الأكردين

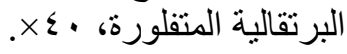

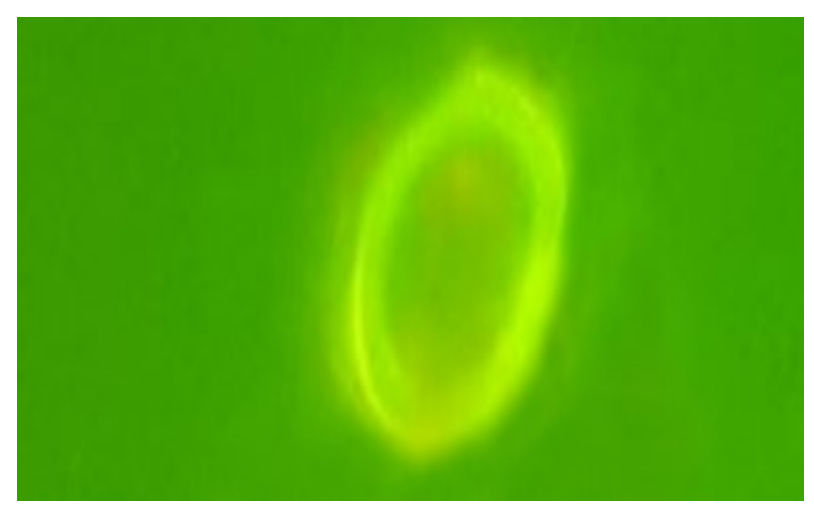

الثكل ب: يوضح بيضة .Ostertagia spp باستخدام صبغة

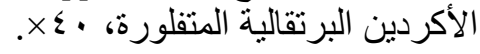

شخصت بيوض الايدان الإسطوانية عند استخدام عدة صبغة

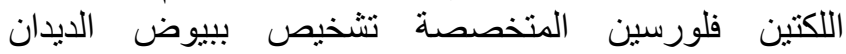
الإسطو انية بهذه التقنية فقد بلغت النسبة الكلية للخمج بهذه بذه الايدان

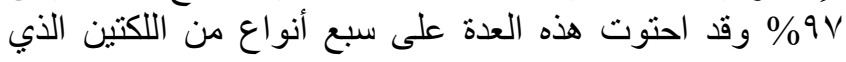




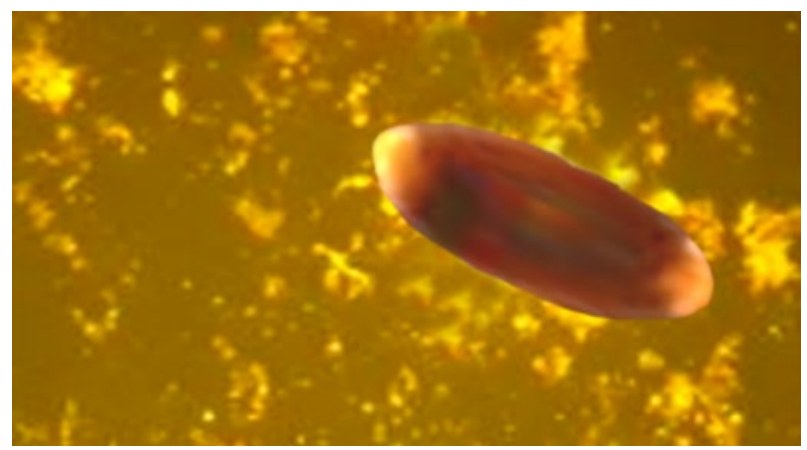

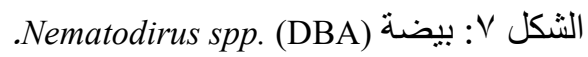

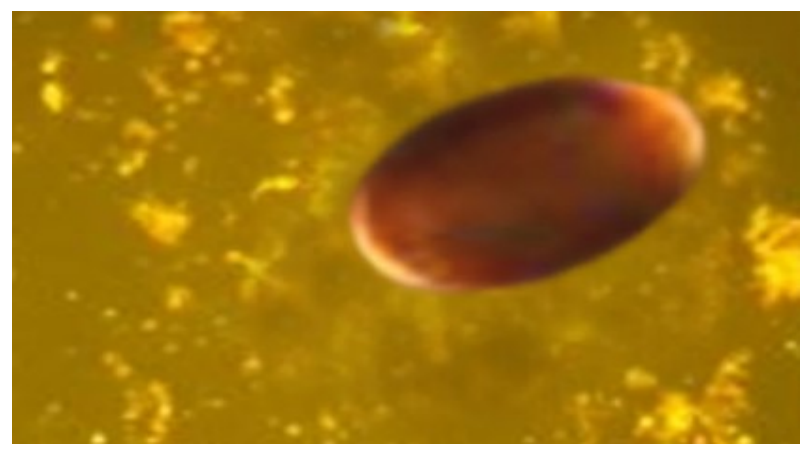

الثكل ؟7: بيضة Trichostrongylus spp. (RCA).

الجدول ا : يبين أنواع اللكتين وشدة ارتباطه مع أنواع بيوض الديدان الإسطو انية

\begin{tabular}{|c|c|c|c|c|}
\hline Chabertia ovina & contotus Haemonchus & Nematodirus spp. & Trichostrongylus spp. & اللكتين ( Lectin) \\
\hline- & - & - & +++ & (Concavaline A) CON A \\
\hline- & - & +++ & - & (Dolichous Biflorus A.) DBA \\
\hline- & +++ & - & - & (Peanut A.) PNA \\
\hline- & - & - & ++ & (Ricinus Comminus A.) RCA \\
\hline- & - & + & - & (Soya Bean A.) SBA \\
\hline- & - & +++ & - & (Ulex Europaeuslec A.) UEA I \\
\hline+++ & - & - & - & (Wheat Germ A.) WGA \\
\hline
\end{tabular}

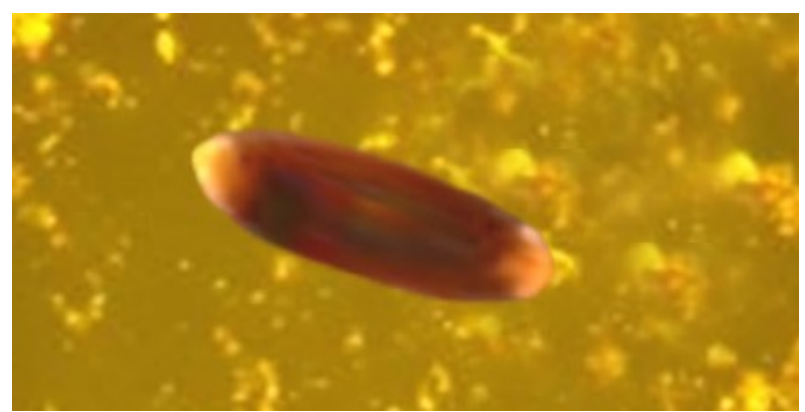

الشكل 9: بيضة Nematodirus spp. (SBA) الشئ

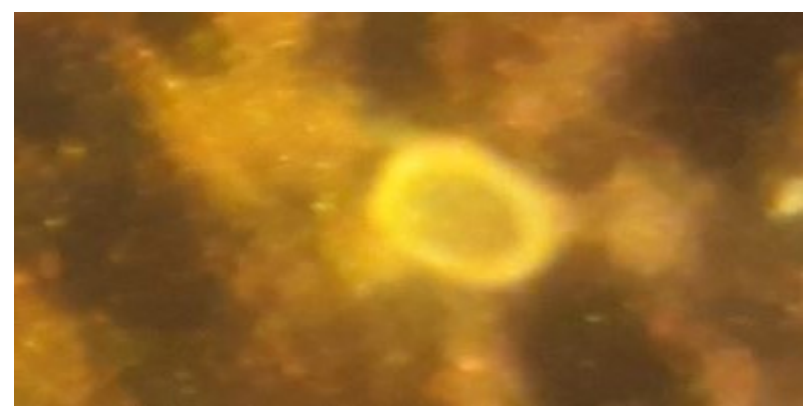

الثكل · ( : بيضة PNA) Haemonchus contotus).
الجدول Y بيين الفرق بين نسبة الخمج بين طريقة التصبيغ بعدة اللكتين - فلورسين وطريقة التصبيغ بصنغة الأكردين البرنقالية

\begin{tabular}{|c|c|c|}
\hline نسبة الخمج بصبغة & نسبة الخمج بعدة & العينات \\
\hline الأكردين البرتقالية & اللكتين - فلورسين & الكلية \\
\hline b\% \% & $a \% 7 \varepsilon$ & 0. \\
\hline
\end{tabular}

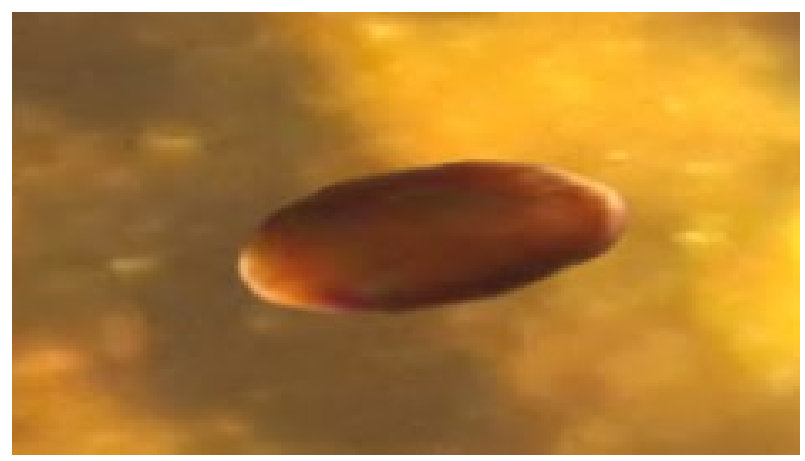

الثكل ^: بيضة Nematodirus spp. (UEA I) - الثل 


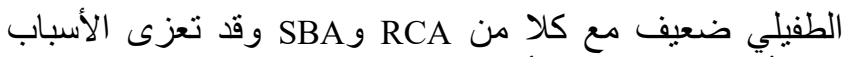

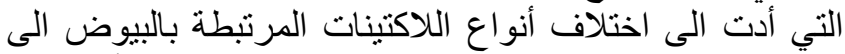

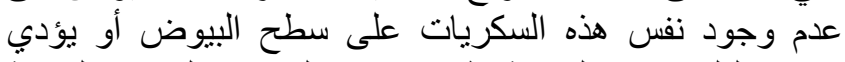

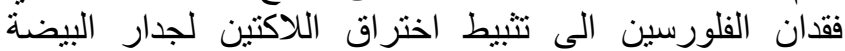

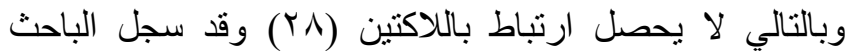
STL II مع (Y) Umaire Nematodirus spp.

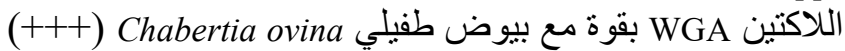

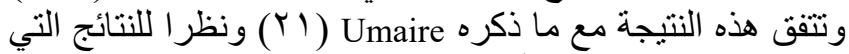
توصلنا اليها نشير اللى أهمية استخدام عدة القيام القياس الجاهزة في

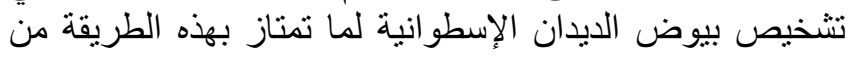

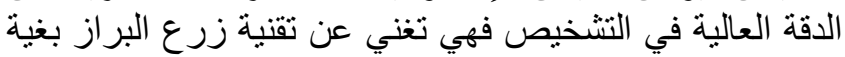

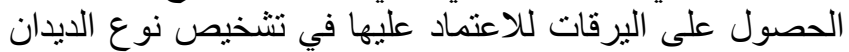
الإسطو انية.

\section{(الاستنتاج}

تمتاز عدة الجاهزة اللكتين - فلورسين بدقتها وكفائتها

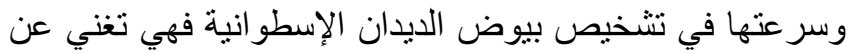

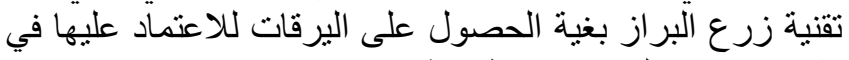
تشخيص نوع الديدان الإسطو انية

\section{الشكر و التقدير}

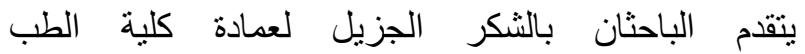

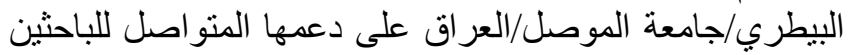

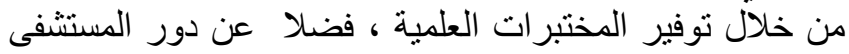

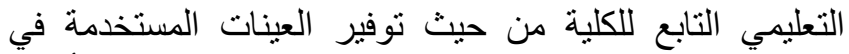

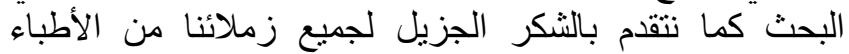

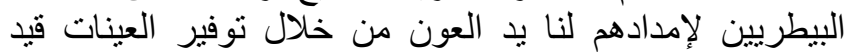

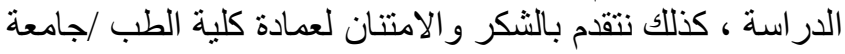

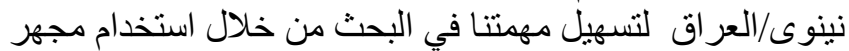

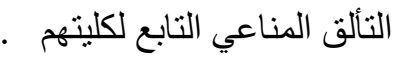

المصادر

1. Bowman DD, Lynn RC, Eberhard ML. Georgi's parasitology for veterinarians. $8^{\text {th }}$ ed. USA: Sanders Elsevier Science; 2003. 153-229p.

2. Gessner T, Mayer U. Triarylmethane and diarylmethane dyes in Ullmann's encyclopedia of industrial chemistry. New York: WileyVCH Weinheim; 2002. http:// ki.se/ sites/ default /files/ gorankronvall as staining mini-review.pdf. doi: 10-1002/14356007.a27179

3. Hemavani N, Chitinis D, Dixit DS, Asolkar MV. Acridine orange stained blood wet mounts for fluorescent detection of malaria. Indian $\mathbf{J}$ Pathol Microbiol. 1999;42:125-128.

4. Keiser J, Utzinger J, Premji Z, Yamagata Y, Singer BH. Acridine orange for malaria diagnosis: Its diagnostic performance, its promotion and implementation in Tanzania, and the implications for

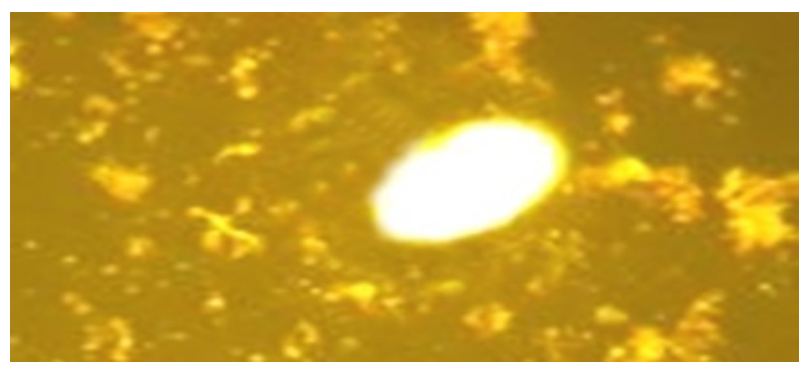

الثكل 11: بيضة Chabertia ovina (WGA).

المناقشة

استخدمت تقنية التصبيغ بصبغة الأكردين البرتقالية حيث الطيث

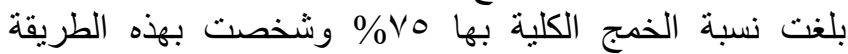
بيوض أجناس متعددة منها. Trichostrongylus spp و و و Bunostomum spp. و Trichuris spp. و spp.

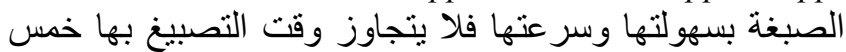

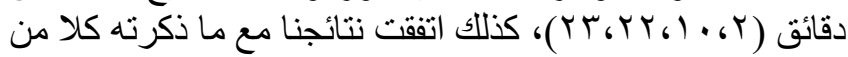
Suleiman and Altaee

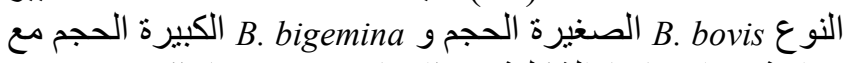

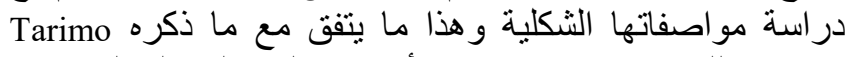

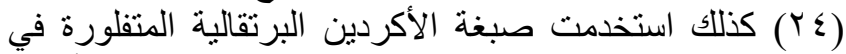

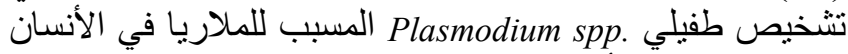

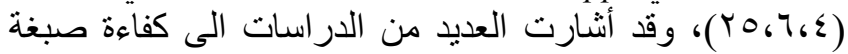

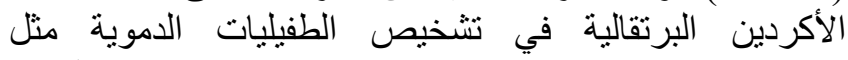
Microfilaria و و M Trypansoma spp.

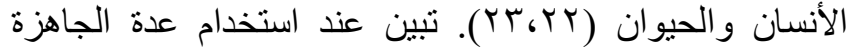

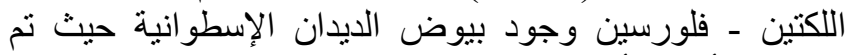
تشخيص أربعة أنواع من بيوض الإن الديدان الإسطوانية المرتبطة الإنة

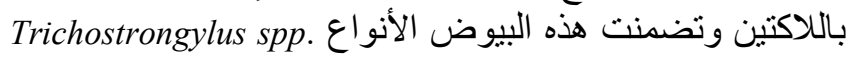

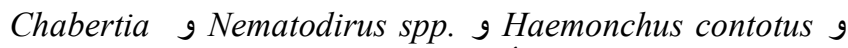
-CON A وشخصت هذه الأنواع بارتباطها باللاكتينات ovina و وعادة برتبط

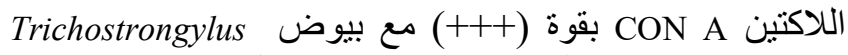
spp. Trichostrongylus spp. Haemonchus contotus

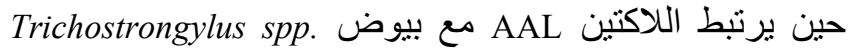

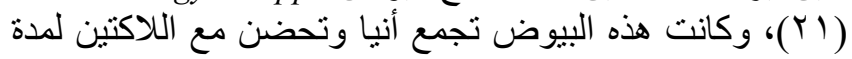

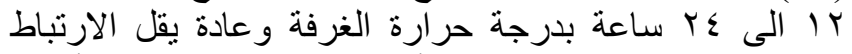

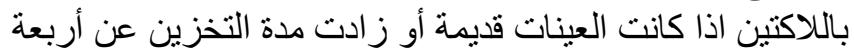

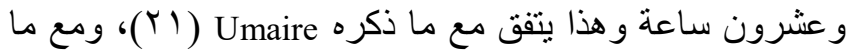

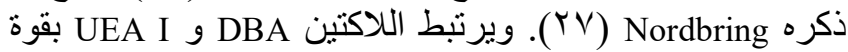

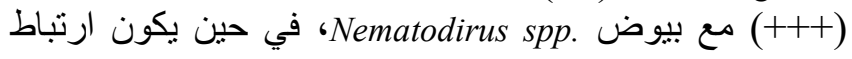


17. Colditz IG, Le LF, Hosse R. Use of lectin binding characteristics and identify gastrointestinal parasite eggs in faeces. Vet Parasitol. 2002;109:219-227doi.org/10.1016/S0304-4017(02)00013-4

18. Foreyt WG. Veterinary parasitology: Reference manual. $5^{\text {th }}$ ed. New York: Blackwell; 2001. 81-91 p.

19. Thienpont D, Rochette F, Vanparijs O. Diagnosing helminthiasis through coprological examination. Belgium: Janssen Foundation Beerse; 1986. 30p.

20. Lauer BA, Reller LB, Mirrett S. Comparison of acridine orange and gram stains for detection of microorganisms in cerebrospinal fluid and other clinical specimens. J Clin Microbiol. 1981;142:201-205.

21. Umair S, McMurtry LW, Knight JS, Simpson HV. Use of fluorescent lectin binding to distinguish eggs of gastrointestinal nematode parasites of sheep. Vet Parasitol. 2015;217:76-80. https://doi.org/10.1016/j.vetpar.2015.12.029

22. Ravindran R, Lakshmanan B, Sreekumar C, Dohn L, Gomathinayagam S, Mishra A, Tewar AK, Raod R. Acridine Orange staining for quick detection of blood parasites. J Vet Parasitol. 2007;211:85-86

23. Goldsmid JM, Rogers S. Preliminary report on the use of acridine orange $\mathrm{O}$ for the detection of Babesia canis in the blood. TST Cent Afr. 1977;232:35-36.

24. Tarimo DS, Mpembeni R, Kawawa H, Mshana TC. Appraisal of the acridine orange method for rapid malaria diagnosis at three Tanzanian district hospital. East Afr Med J. 1998;75:504-507.

25. Gay F, Boubacar T, Zanoni D, Danis M, Balnc AF. Direct acridine orange fluorescence examination of blood slides compared to current techniques for malaria diagnosis. Trans $\mathrm{R}$ Soc Trop Med Hyg. 1996;90:516-518.

26. Hillrichs K, Schnieder T, Forbes AB, Simcock DC, Pedley K, Simpson HV. Use of fluorescent lectin binding to distinguish Teladorsagia circumcincta and Haemonchus contortus eggs third stage larvae and adult worm. Parasitol Res. 2012;110:449-458.

27. Nordbring B. Action of a nematode-trapping fungus shows lectinmediated host-microorganism interaction. Nature. 1979;281:477-479.

28. Chaney A. Identification of internal parasites of sheep and goat [MSc thesis]. Houston: Honor College; 2012. 26 p. malaria control. Ann Trop Med Parasiotol. 2002;957:643-654. DOI: $10.1179 / 000349802125001834$

5. Gainer JH. Demonstration of Anaplasma marginale with the fluorescent dye, acridine orange, comparisons with the complement fixation test and wright's stain. Am J Vet Res. 1961;22;882-886.

6. Kawamoto F. Rapid diagnosis of malaria by Fluorescence microscopy. Lancet. 1991; 337:200-202.doi.org/10.1016/01406736(91)92159-Y

7. Fripp PD, Mason PR, Super HA. Method for the detection of Trichomonas vaginalis using acridine orange. $\mathrm{J}$ Parasitol. 1975;61:966-967.

8. Levett PN. A comparison of five methods for the detection of Trichomonas vaginalis in clinical specimens. Med Lab Sci. 1980;37:85-88.

9. Shute GT, Sodeman TM. Identification of malaria parasites by fluorescence microscopy and acridine orange staining. Bull World Heal Org. 1973;485:591-596.

10. Kong HH, Chung D. Comparison of acridine orange and giemsa stains for malaria diagnosis. Korean J Parasitol. 1995;334:391-394.

11. Yoon E, Vail E, Sann L, Brass J. New staining technique for diagnosing Babesia species. Amer J Clin Pathol. 2015;144(Suppl 2,1):228. doi.org/10.1093/ajcp/144.supp12.228

12. Salih DA, El Hussein AM, Singla LD. Diagnostic approaches for tickborne haemoparastic diseases in livestock. J Vet Med Anim Heal. 2015;72:45-56.DOI:10.5897/JVMAH2014.0345.

13. Suleiman EG. Morphological and molecular study of Babesia spp. and isolation and diagnosis of tick vector in infected cattle in Mosul city [PhD dissertation]. Mosul: University of Mosul, College of Veterinary Medicine; 2018. 124-126 p.

14. Suleiman EG, Altaee AF. The possibility of using acridine orange compared to giemsa stain in the diagnosis of parasite Babesia spp. in $\begin{array}{lllll}\text { cattle. J Vet } & \text { Med } & \text { Sci. 2019;33(2):1-7. }\end{array}$ doi.org/10.33899/ijvs.2019.153870

15. Silvestre A, Humbert JF. A molecular tool for species identification and benzimidazole resistance diagnosis in larval communities of small ruminant parasites. Exp Parasitol. 2000;95:271-276. doi.org/10.1006/expr.2000.4542

16. Hamid R, Masood A. A dietary lectin as disease causing toxicants. Pakistan J Nutri. 2009;3:293-303. 